ISSN 1991-8631

\title{
Etude comparative des teneurs en polyphénols et en antioxydants totaux d'extraits de graines de 44 variétés de voandzou (Vigna subterranea (L.)Verdcourt)
}

\author{
Abel MBAIOGAOU ${ }^{1}$, Adama HEMA ${ }^{1 *}$, Mahama OUÉDRAOGO ${ }^{2}$, Eloi PALÉ ${ }^{1}$, \\ Michel NAITORMBAIDE ${ }^{3}$, Yaya MAHAMOUT ${ }^{4}$ et Mouhoussine NACRO $^{1}$ \\ ${ }^{1}$ Laboratoire de Chimie Organique et de Physique Appliquées, Département de Chimie, UFR-SEA, \\ Université de Ouagadougou; 03 BP 7021 Ouagadougou 03, Burkina Faso. \\ ${ }^{2}$ Institut de l'Environnement et de Recherches Agricoles (INERA), Ouagadougou, Burkina Faso. \\ ${ }^{3}$ Institut Tchadien de Recherche Agronomique pour le Développement (ITRAD) de Bebedja, Tchad. \\ ${ }^{4}$ Laboratoire de Recherche sur les Substances Naturelles, Faculté des Sciences Exactes et Appliquées (F.S.E.A) \\ de l'Université de N'Djamena, Tchad. \\ *Auteur correspondant; E-mail: hemaadama@yahoo.fr
}

\section{RESUME}

44 variétés de Vigna subterranea provenant de l'Institut de l'Environnement et de Recherches Agricoles, au Burkina Faso et de l'Institut Tchadien de Recherche Agricole pour le Développement au Tchad, ont fait l'objet d'une étude comparative de leurs teneurs en polyphénols et en antioxydants totaux. Les teneurs en polyphénols totaux ont été déterminées par la méthode utilisant le réactif du Folin-Ciocalteu et les teneurs en antioxydants ont été évaluées par trois méthodes courantes, simples et disponibles: Trolox Equivalents Antioxidant Capacity (TEAC), 1,1-Diphenyl-1-picrylhydrazyl (DPPH) et Ferric Reducing Antioxidant Power (FRAP). L'étude a montré que les polyphénols, responsables de la couleur des péricarpes contribuent à $94 \%$ à la teneur en antioxydants totaux des extraits étudiés. Ainsi, à travers les comparaisons effectuées, les 17 variétés suivantes ont été sélectionnées comme étant les plus riches en polyphénols $(\leq 4,536 \mathrm{mg}$ d'EAG/g) et en antioxydants totaux ( $\leq 10,72 \mathrm{mg}$ d'ET/g; FRAP) : KVS350, KVS314, M2, D2, KVS311, M3, KVS109, KVS67, KVS288, KVS153, KVS360, M4, KVS141, M7, KVS225, KVS97, KVS312 (P<0,001). Ces variétés possèdent des teneurs en polyphénols totaux plus élevées que celles de Vicia faba/faba vulgaris, Phaseolus vulgaris, vigna unguiculata et d'autres légumineuses de la même famille des Fabaceae.

(C) 2013 International Formulae Group. All rights reserved.

Mots clés: Fabaceae, TEAC, DPPH, FRAP, Folin-Ciocalteu, anthocyanes totales.

\section{INTRODUCTION}

La plupart des aliments de l'homme proviennent du règne végétal. Ces produits naturels apportent à l'homme des macronutriments tels que les matières grasses, les sucres rapides, les sucres lents et les protéines dont il a besoin. Outre ces macronutriments énergétiques bien connus dans les aliments traditionnels, il existe d'autres substances dites secondaires (micronutriments) qui sont de plus en plus populaires pour leurs effets bénéfiques sur la santé des consommateurs. 
Les légumineuses du genre Vigna font partie des aliments les plus consommés en Afrique au sud du Sahara (Brink et al., 2006). Connu sous les noms courants «voandzou» en français, « souma » en mooré (au Burkina Faso) et «oule-ndâ » en ngambaye (au Tchad), le voandzou est la deuxième légumineuse économiquement importante après le niébé [Vigna unguiculata (L.) Walpers] dans les pays au sud du Sahara. C'est une plante qui s'adapte bien aux conditions climatiques difficiles (Heller et al., 1997). Les graines sont très nutritives et les analyses chimiques ont montré qu'elles contiennent $32.72 \%$ d'acides aminés totaux essentiels et $66.10 \%$ d'aminoacides totaux non-essentiels (Minka et al., 2000 ; Mahama et al., 2008 ). D'autres légumineuses du même genre sont reconnues renfermer des micronutriments responsables des couleurs observées au niveau des graines. Ces micronutriments, généralement antioxydants, sont très abondants dans les fruits et les légumes et interviennent dans la prévention des cancers, des maladies cardio-vasculaires et d'autres maladies dégénératives liées au stress oxydant (Navindra, 2008 ; Yanjun et al., 2008). Dans les pays au sud du Sahara où les légumineuses du genre Vigna notamment le $V$. subterranea sont beaucoup consommées, il convient d'avoir une base de données sur leurs teneurs en antioxydants qui informe les consommateurs sur leurs effets bénéfiques sur la santé (Liangli et al., 2005). En effet, ces données sur leurs teneurs en antioxydants totaux constituent des informations nutritionnelles supplémentaires qui faciliteront une meilleure intégration du voandzou parmi les aliments locaux à caractère fonctionnel à l'instar d'autres fruits et légumes (Liu et al., 2003; Jimaima et al., 2007).

Plusieurs méthodes ont été fréquemment utilisées pour évaluer les activités antioxydantes dans les extraits végétaux. On peut entre autres citer le DPPH (1,1-diphenyl-1-picrylhydrazyl), l'ABTS (2,2'-azinobis (3-ethylbenzothiazoline-6sulfonique), et le FRAP (ferric reducing antioxidant power). Ces techniques ont montré des résultats différents des échantillons de plante testés à travers les laboratoires. Le réactif de Folin-Ciocalteu est utilisé pour évaluer les teneurs en polyphénols totaux et la méthode du pH-différentiel est utilisée pour évaluer les teneurs en anthocyanes totales.

L'objectif de la présente étude était de déterminer les teneurs en polyphénols et en anthocyanes totaux et d'estimer les teneurs en antioxydants totaux en utilisant les méthodes de DPPH, ABTS, et FRAP (Stephanie et al., 2009) de 44 variétés de voandzou du Burkina Faso et du Tchad. Ces trois méthodes basées sur différents mécanismes chimiques ont été choisies pour prendre en compte la large variabilité et de gamme d'action des antioxydants individuels des extraits étudiés. Le TEAC mesure la capacité des antioxydants à piéger le radical-cation $\left(A B T S^{+*}\right)$ (Pellegrini et al., 2003), le DPPH mesure la capacité de piégeage du radical stable commercial $_{\left(\text {DPPH }^{-}\right)}$) (Brand-Williams et al., 1995) et le FRAP, une méthode directe qui évalue le pouvoir réducteur d'un échantillon (Benzie et al., 1996).

\section{MATÉRIEL ET MÉTHODES \\ Matériel végétal}

L'étude porte sur 30 variétés de $V$. subterranea du Burkina Faso et quatorze variétés du Tchad récoltées en fin octobre. Ces différentes variétés sont fournies respectivement par INERA de Ouagadougou (Burkina Faso) et ITRAD de Deli (Tchad).

\section{Extraction}

Les graines des différentes variétés de $V$. subterranea ont été broyées. Ensuite, $3 \mathrm{~g}$ de poudre de chaque variété sont extraits avec 15 $\mathrm{mL}$ du système acétone-eau-acide acétique (70: 29,5:0,5) par macération pendant 24 heures à $4{ }^{\circ} \mathrm{C}$. Les extraits sont filtrés et les résidus sont extraits de nouveau deux fois avec $10 \mathrm{~mL}$ de solvant pendant 24 heures. Les filtrats sont conservés au réfrigérateur à $4{ }^{\circ} \mathrm{C}$ pour la détermination des teneurs des 
polyphénols et des antioxydants totaux (Asami et al., 2003).

\section{Réactif de Folin-Ciocalateu}

Les teneurs en polyphénols totaux des extraits de voandzou ont été déterminées par la méthode de Folin-Ciocalteu (Nihal et al., 2007). Elle consiste à faire réagir $60 \mu \mathrm{L}$ de l'échantillon étudié avec $60 \mu \mathrm{L}$ du réactif de Folin (dilué 10 fois). Après $8 \mathrm{~min}, 120 \mu \mathrm{L}$ de carbonate de sodium à $7,5 \%(\mathrm{p} / \mathrm{v})$ y sont ajoutés. Après $30 \mathrm{~min}$ d'incubation, l'absorbance est lue à $765 \mathrm{~nm}$. Les blancs sont préparés pour chaque variété en remplaçant le réactif de Folin par de l'eau distillée. L'acide gallique est utilisé comme standard (Tableau 1) et les résultats sont exprimés en mg d'équivalents d'acide gallique (EAG)/g de matériel sec.

\section{pH différentiel}

Les teneurs en anthocyanes totales des extraits sont estimées par la méthode de $\mathrm{pH}$ différentiel utilisant deux systèmes tampon: la solution de chlorure de potassium, $\mathrm{pH}$ 1,0 $(0,025 \mathrm{M})$ et la solution d'acétate, $\mathrm{pH} 4,5$ $(0,025 \mathrm{M}) .100 \mu \mathrm{L}$ de l'extrait sont mélangés à $200 \mu \mathrm{L}$ des tampons correspondants et l'absorbance est lue par rapport au blanc à 510 $\mathrm{nm}$ et à $700 \mathrm{~nm} 15$ minutes plus tard. L'absorbance A a été calculée comme suit : $\mathrm{A}=\left(\mathrm{A}_{510}-\mathrm{A}_{700}\right)_{\mathrm{pH} 1.0}-\left(\mathrm{A}_{510}-\mathrm{A}_{700}\right)_{\mathrm{pH} 4.5}$ La concentration monomérique en colorants anthocyaniques dans l'extrait est calculée comme la cyanidin-3-glucoside (Sellapan et al., 2002).

$$
\left(m g / L=\frac{A \times P M \times F D \times 1000}{\varepsilon \times l}\right)
$$

où $\mathrm{A}$ : absorbance ; PM : poids moléculaire ; $(449,2)$; FD : facteur de dilution ; $\varepsilon$ : absorptivité molaire (26900). Les teneurs en anthocyanes totales ont été exprimées en microgrammes de cyanidin-3-glucoside par gramme de matériel sec.

\section{Méthode du TEAC}

Le radical-cation ABTS est généré en mélangeant $1 \mathrm{~mL}$ de solution à $39,2 \mathrm{mM}$ de persulfate de potassium $\mathrm{K}_{2} \mathrm{~S}_{2} \mathrm{O}_{8}$ et $5 \mathrm{~mL}$ de solution d'ABTS à 7,01 mM, le mélange est conservé à l'abri de la lumière à la température de $4{ }^{0} \mathrm{C}$ durant 16 heures, temps nécessaire pour la génération du radicalcation. La solution bleu-vert obtenue est diluée pour avoir une absorbance de $0,7 \pm 0,5$ à $734 \mathrm{~nm}$. À chaque échantillon $(50 \mu \mathrm{L})$ sont ajoutés $200 \mu \mathrm{L}$ de solution de ABTS et l'absorbance est mesurée après 10 min à 734 $\mathrm{nm}$. Le résultat est exprimé en mg d'Equivalents de Trolox (ET) (Tableau 1) (Pellegrini et al., 2003).

\section{Méthode de DPPH}

Le radical commercial de DPPH est dissout dans du méthanol à une concentration de $0,04 \mathrm{mg} / \mathrm{mL}$ et gardé à $4{ }^{\circ} \mathrm{C}$ à l'abri de la lumière. À chaque échantillon d'extrait (50 $\mu \mathrm{L}$ ) sont ajoutés $200 \mu \mathrm{L}$ de solution de DPPH et l'absorbance est lue après $10 \mathrm{~min}$ à $517 \mathrm{~nm}$. Les résultats sont exprimés en mg d'ET (Tableau 1) (Stephanie et al., 2009).

\section{Méthode du FRAP}

Le réactif de la méthode du FRAP (TPTZ) est obtenu en mélangeant une solution de TPTZ (10 $\mathrm{nM})$, une solution tampon d'acétate de sodium $(\mathrm{pH} 3,6)$ et une solution $\mathrm{FeCl}_{3}(20 \mathrm{nM})$ dans les proportions 1:10:1. À chaque échantillon d'extrait $(20 \mu \mathrm{L})$ sont ajoutés $30 \mu \mathrm{L}$ d'eau et $200 \mu \mathrm{L}$ de solution de FRAP et l'absorbance est mesurée après 10 min à $595 \mathrm{~nm}$. Les résultats est exprimés en mg d'ET (Tableau 1) (Benzie et al., 1996 ; Proteggente et al., 2002).

\section{Étude statistique}

L'étude statistique a été réalisée par le logiciel statistique GentStat $14^{\mathrm{e}}$ Edition au seuil de probabilité de 5\%. Toutes les expériences ont été réalisées en triple. Les résultats sont exprimés sous la forme moyenne \pm écart type. Les valeurs de $p<0,05$ sont considérées statistiquement significatives (Athamena et al., 2010). 
Tableau 1 : Etablissement des courbes-étalons.

\begin{tabular}{lccc}
\hline Courbes-étalons & Standard & Equations & coefficients de corrélation \\
\hline RFC & Acide gallique & $\mathrm{y}=43,57 \mathrm{x}+0,200$ & $\mathrm{R}^{2}=0,990$ \\
ABTS (TEAC) & Trolox & $\mathrm{y}=-23,36 \mathrm{x}+0,590$ & $\mathrm{R}^{2}=0,999$ \\
DPPH & Trolox & $\mathrm{y}=-26,99 \mathrm{x}+0,636$ & $\mathrm{R}^{2}=0,999$ \\
FRAP & Trolox & $\mathrm{y}=25,47 \mathrm{x}+0,068$ & $\mathrm{R}^{2}=1$ \\
\hline
\end{tabular}

\section{RESULTATS}

Il a été systématiquement déterminé les teneurs en antioxydants, en polyphénols et en anthocyanes totaux de 44 variétés de voandzou provenant du Burkina Faso et du Tchad. Ces 44 variétés sont caractérisées par la couleur jaune-crème, noire pourpre ou bigarrée de leur péricarpe. Les différences entre les teneurs en antioxydants, en polyphénols et en anthocyanes totaux (Tableau 2) de ces variétés de voandzou sont souvent très larges. Cette variation des teneurs est probablement liée à la couleur de ces graines.

\section{Teneurs en antioxydants totaux (TAO)}

En se basant sur les teneurs en antioxydants, certaines variétés de voandzou présentent des valeurs très différentes. En effet, les trois méthodes utilisées pour déterminer les TAO (DPPH, TEAC et FRAP) révèlent des variations respectives d'environ 28 fois, 13 fois et 50 fois. Par la méthode de DPPH (Tableau 2 ; Figure 1), il ressort que les variétés KVS416, KVS411, KVS358 ont pratiquement la même teneur en antioxydants d'environ $0,072 \mathrm{mg}$ d'ET/g de graines sèches (les différences observées ne sont pas statistiquement significatives). Ces dernières sont moins riches que les variétés KVS358, KVS202, KVS415 présentant une teneur en antioxydants d'environ $0,171 \mathrm{mg}$ d'ET/g de graines sèches. Par cette méthode, les plus riches sont les variétés M4, KVS312, KVS97 présentant une teneur d'environ 2,039 mg d'ET/g de graines sèches. Il en résulte donc que les teneurs en antioxydants varient largement entre 0,072 et 2,039 mg d'ET/g de graines sèches.

Par la méthode de TEAC, il résulte des différences très significatives $(P<0,001)$ entre les différentes variétés de graines de $V$. subterranea. En effet, les TAO varient de 0,17 pour la variété KVS411 à 2,327 mg d'ET/g de graines sèches pour les variétés M4, M7 (Tableau 1, Figure 2) soit une variation d'environ 13 fois. Par ailleurs, on note une assez bonne corrélation (Tableau 3 ; Figure 4) entre ces deux méthodes $(R=0,964)$; ce qui montre que ces deux méthodes, ne divergent pas fondamentalement dans l'explication de la variabilité constatée entre ces variétés de $V$. subterranea.

La troisième méthode, beaucoup plus directe que les deux précédentes, la méthode FRAP est basée sur la réduction des ions ferriques en ions ferreux par les antioxydants des extraits. Elle contribue à expliquer les différences significatives entre les variétés de $V$. subterranea. Ainsi, on observe une variation d'environ 50 fois soit des teneurs variant entre 0,214 pour les variétés KVS411, KVS358, KVS202, KVS415, KVS416, KVS199 à 10,727 mg d'ET/g de graines sèches pour la variété KVS225 qui en est la plus riche (Tableau 2 et Figure 3). Par ailleurs, cette méthode présente d'assez bonnes corrélations $(\mathrm{R}=0,934 ; \mathrm{R}=0,939)$ respectivement avec les méthodes DPPH et TEAC (Tableau 3). 
Tableau 2: Teneurs en antioxydants (TAO) (par les méthodes de DPPH, TEAC et FRAP), en polyphénols totaux (TPP) et en anthocyanes totales (TAT) dans les différentes variétés des graines de V. subterranea.

\begin{tabular}{|c|c|c|c|c|c|}
\hline \multirow{2}{*}{ variétés } & \multicolumn{3}{|c|}{ TAO (mg/g) } & \multirow{2}{*}{$\begin{array}{c}\text { TPP (mg/g) } \\
\text { RFC }\end{array}$} & \multirow{2}{*}{$\begin{array}{c}\text { TAT (mg/g) } \\
\text { pH-diff. }\end{array}$} \\
\hline & DPPH & TEAC & FRAP & & \\
\hline KVS141 & $1,921 \pm 0,019 u v w$ & $1,923 \pm 0,003 \mathrm{t}$ & $8,593 \pm 0,021 \mathrm{~s}$ & $3,736 \pm 0,051$ op & $0,053 \pm 0,001 \mathrm{r}$ \\
\hline KVS109 & $1,793 \pm 0,021 \mathrm{t}$ & $1,795 \pm 0,005 \mathrm{r}$ & $6,701 \pm 0,155 n$ & $2,828 \pm, 045 \mathrm{n}$ & $0,012 \pm 0,0008 \mathrm{cdefg}$ \\
\hline KVS61 & $0,6485 \pm 0,003 \mathrm{ij}$ & $0,656 \pm 0,003 \mathrm{fgh}$ & $1,215 \pm 0,008 \mathrm{de}$ & $0,405 \pm 0,008 \mathrm{de}$ & $0,017 \pm 0,0004 \mathrm{hijk}$ \\
\hline KVS416 & $0,071 \pm 0,005 a$ & $0,411 \pm 0,016 \mathrm{~d}$ & $0,442 \pm 0,015 \mathrm{a}$ & $0,125 \pm 0,014 \mathrm{abc}$ & $0,004 \pm 0,0005 \mathrm{a}$ \\
\hline KVS407 & $0,595 \pm 0,018 \mathrm{hi}$ & $0,667 \pm 0,008 \mathrm{gh}$ & $0,938 \pm 0,030 \mathrm{~cd}$ & $0,272 \pm 0,006 \mathrm{bcd}$ & $0,015 \pm 0,0009$ efghij \\
\hline KVS350 & $1,581 \pm 0,009 \mathrm{r}$ & $1,474 \pm 0,016$ op & $5,101 \pm 0,0901$ & $1,86 \pm 0,072 \mathrm{k}$ & $0,161 \pm 0,004 \mathrm{t}$ \\
\hline KVS225 & $1,906 \pm 0,002 \mathrm{w}$ & $1,842 \pm 0,007 \mathrm{rs}$ & $10,727 \pm 0,043 \mathrm{v}$ & $4,406 \pm 0,055 \mathrm{q}$ & $0,034 \pm 0,001$ no \\
\hline KVS311 & $1,677 \pm 0,010 \mathrm{~s}$ & $1,63 \pm 0,026 \mathrm{q}$ & $6,027 \pm 0,184 \mathrm{~m}$ & $2,51 \pm 0,067 \mathrm{~m}$ & $0,011 \pm 0,0005$ bcdef \\
\hline KVS314 & $1,402 \pm 0,013$ o & $1,404 \pm 0,011 \mathrm{mn}$ & $5,018 \pm 0,0141$ & $2,284 \pm 0,0211$ & $0,02 \pm 0,0009 \mathrm{klm}$ \\
\hline KVS358 & $0,13 \pm 0,023 \mathrm{abc}$ & $0,313 \pm 0,006 b c$ & $0,37 \pm 0,007 \mathrm{a}$ & $0,052 \pm 0,006 \mathrm{a}$ & $0,015 \pm 0,0003$ efghij \\
\hline KVS32 & $1,43 \pm 0,009$ op & $1,677 \pm 0,035 \mathrm{q}$ & $4,276 \pm 0,0075 \mathrm{jk}$ & $1,554 \pm 0,072 \mathrm{j}$ & $0,041 \pm 0,0014 \mathrm{pq}$ \\
\hline KVS415 & $0,171 \pm 0,015 \mathrm{c}$ & $0,37 \pm 0,025 \mathrm{~cd}$ & $0,419 \pm 0,009 \mathrm{a}$ & $0,102 \pm 0,013 \mathrm{ab}$ & $0,007 \pm 0,0001 \mathrm{abc}$ \\
\hline KVS120 & $1,262 \pm 0,011 \mathrm{n}$ & $1,227 \pm 0,0151$ & $3,148 \pm 0,042 \mathrm{i}$ & $1,068 \pm 0,001 \mathrm{~h}$ & $0,03 \pm 0,0007 \mathrm{n}$ \\
\hline KVS360 & $1,836 \pm 0,007 \mathrm{tu}$ & $1,867 \pm 0,005 \mathrm{st}$ & $9,233 \pm 0,219 \mathrm{t}$ & $3,595 \pm 0,006$ о & $0,034 \pm 0,0008$ no \\
\hline KVS128 & $0,475 \pm 0,002 \mathrm{ef}$ & $0,586 \pm 0,009 \mathrm{e}$ & $0,855 \pm 0,017 \mathrm{c}$ & $0,156 \pm 0,015 \mathrm{abc}$ & $0,006 \pm 0,0007 \mathrm{a}$ \\
\hline KVS199 & $0,409 \pm 0,004 \mathrm{~d}$ & $0,374 \pm 0,012 \mathrm{~cd}$ & $0,520 \pm 0,016 \mathrm{ab}$ & $0,154 \pm 0,002 \mathrm{abc}$ & $0,018 \pm 0,0005 \mathrm{ijkl}$ \\
\hline KVS97 & $2,038 \pm 0,002 \times$ & $2,242 \pm 0,010 \mathrm{w}$ & $8,44 \pm 0,010 \mathrm{rs}$ & $4,533 \pm 0,128 \mathrm{q}$ & $0,331 \pm 0,0023 \mathrm{v}$ \\
\hline KVS153 & $1,903 \pm 0,016 \mathrm{vw}$ & $2,107 \pm 0,006 \mathrm{v}$ & $7,408 \pm 0,081 \mathrm{pq}$ & $2,997 \pm 0,051 \mathrm{n}$ & $0,01 \pm 0,0009$ bcde \\
\hline KVS288 & $1,883 \pm 0,016 u v w$ & $2,053 \pm 0,021 \mathrm{uv}$ & $6,929 \pm 0,101$ no & $2,964 \pm 0,014 \mathrm{n}$ & $0,03 \pm 0,000008 \mathrm{n}$ \\
\hline KVS351 & $0,422 \pm 0,020 \mathrm{de}$ & $0,633 \pm 0,015 \mathrm{efg}$ & $1,024 \pm 0,025 \mathrm{~cd}$ & $0,316 \pm 0,008 \mathrm{~cd}$ & $0,008 \pm 0,0002 \mathrm{abc}$ \\
\hline KVS210 & $0,58 \pm 0,035 \mathrm{~h}$ & $0,706 \pm 0,006 \mathrm{hi}$ & $1,148 \pm 0,012 \mathrm{cde}$ & $0,405 \pm 0,013 \mathrm{de}$ & $0,017 \pm 0,0008$ ghijk \\
\hline KVS312 & $2,006 \pm 0,021 \mathrm{x}$ & $2,239 \pm 0,002 \mathrm{w}$ & $8,466 \pm 0,013 \mathrm{rs}$ & $4,536 \pm 0,089 \mathrm{q}$ & $0,03 \pm 0,0002 \mathrm{n}$ \\
\hline KVS67 & $1,839 \pm 0,006$ tuv & $2,038 \pm 0,004 \mathrm{u}$ & $7,223 \pm 0,092$ op & $2,876 \pm 0,027 \mathrm{n}$ & $0,012 \pm 0,0007 \mathrm{cdef}$ \\
\hline M1 & $1,42 \pm 0,003$ op & $1,663 \pm 0,005 \mathrm{q}$ & $3,197 \pm 0,031 \mathrm{i}$ & $1,101 \pm 0,024 \mathrm{hi}$ & $0,037 \pm 0,0012$ op \\
\hline KVS41 & $0,851 \pm 0,009 \mathrm{kl}$ & $1,042 \pm 0,031 \mathrm{k}$ & $1,715 \pm 0,047 \mathrm{fg}$ & $0,67 \pm 0,006 f g$ & $0,042 \pm 0,0009 \mathrm{q}$ \\
\hline KVS408 & $1,208 \pm 0,009 \mathrm{n}$ & $1,369 \pm 0,011 \mathrm{~m}$ & $3,043 \pm 0,088 \mathrm{i}$ & $1,183 \pm 0,009 \mathrm{hi}$ & $0,008 \pm 0,0006 a b c$ \\
\hline KVS411 & $0,093 \pm 0,004 \mathrm{ab}$ & $0,17 \pm 0,011 \mathrm{a}$ & $0,213 \pm 0,010 \mathrm{a}$ & $0,046 \pm 0,004 \mathrm{a}$ & $0,007 \pm 0,0005 \mathrm{ab}$ \\
\hline M4 & $1,991 \pm 0,009 \mathrm{x}$ & $2,308 \pm 0,002 \times$ & $8,154 \pm 0,085 \mathrm{r}$ & $3,673 \pm 0,089$ о & $0,281 \pm 0,0015 \mathrm{u}$ \\
\hline KVS202 & $0,142 \pm 0,020 \mathrm{bc}$ & $0,253 \pm 0,010 \mathrm{~b}$ & $0,379 \pm 0,013 \mathrm{a}$ & $0,193 \pm 0,006 \mathrm{abc}$ & $0,005 \pm 0,0002 \mathrm{a}$ \\
\hline KVS410 & $0,918 \pm 0,012 \mathrm{~m}$ & $1,035 \pm 0,020 \mathrm{k}$ & $1,772 \pm 0,025 \mathrm{~g}$ & $0,599 \pm 0,006 \mathrm{ef}$ & $0,024 \pm 0,0006 \mathrm{~m}$ \\
\hline KVS310 & $0,497 \pm 0,030 \mathrm{fg}$ & $0,601 \pm 0,017$ ef & $0,837 \pm 0,009 b c$ & $0,285 \pm 0,005 \mathrm{bcd}$ & $0,022 \pm 0,0020 \mathrm{~lm}$ \\
\hline KVS414 & $0,556 \pm 0,014 \mathrm{gh}$ & $0,634 \pm 0,008 \mathrm{efg}$ & $0,944 \pm 0,023 \mathrm{~cd}$ & $0,278 \pm 0,003 \mathrm{bcd}$ & $0,009 \pm 0,0006 \mathrm{abcd}$ \\
\hline D2 & $1,489 \pm 0,011 \mathrm{pq}$ & $2,013 \pm 0,012 \mathrm{u}$ & $7,616 \pm 0,084 \mathrm{q}$ & $2,386 \pm 0,054 \mathrm{~lm}$ & $0,016 \pm 0,0002$ fghijk \\
\hline D7 & $0,802 \pm 0,0004 \mathrm{k}$ & $0,832 \pm 0,010 \mathrm{j}$ & $3,348 \pm 0,026 \mathrm{i}$ & $0,829 \pm 0,023 \mathrm{~g}$ & $0,014 \pm 0,0016$ efghi \\
\hline D4 & $0,671 \pm 0,003 \mathrm{j}$ & $0,414 \pm 0,004 \mathrm{~d}$ & $1,449 \pm 0,002 \mathrm{ef}$ & $0,286 \pm 0,013 \mathrm{bcd}$ & $0,024 \pm 0,0003 \mathrm{~m}$ \\
\hline D6 & $1,254 \pm 0,003 n$ & $1,369 \pm 0,001 \mathrm{~m}$ & $4,093 \pm 0,025 \mathrm{j}$ & $1,304 \pm 0,035 \mathrm{i}$ & $0,017 \pm 0,0007$ ghijk \\
\hline D1 & $0,893 \pm 0,024 \mathrm{~lm}$ & $0,744 \pm 0,012 \mathrm{i}$ & $2,133 \pm 0,013 \mathrm{~h}$ & $0,559 \pm 0,006 \mathrm{ef}$ & $0,036 \pm 0,0003$ o \\
\hline D3 & $1,229 \pm 0,013 \mathrm{n}$ & $1,459 \pm 0,009$ no & $4,527 \pm 0,036 \mathrm{k}$ & $1,598 \pm 0,009 \mathrm{j}$ & $0,139 \pm 0,0005 \mathrm{~s}$ \\
\hline D5 & $0,865 \pm 0,007 \mathrm{klm}$ & $0,865 \pm 0,002 \mathrm{j}$ & $1,998 \pm 0,035 \mathrm{gh}$ & $0,6 \pm 0,009 \mathrm{ef}$ & $0,019 \pm 0,0002 \mathrm{jkl}$ \\
\hline M7 & $1,65 \pm 0,001 \mathrm{~s}$ & $2,326 \pm 0,003 x$ & $9,721 \pm 0,079 \mathrm{u}$ & $3,913 \pm 0,083$ op & $0,03 \pm 0,0009 \mathrm{n}$ \\
\hline M2 & $1,548 \pm 0,001 \mathrm{r}$ & $2,071 \pm 0,009 \mathrm{uv}$ & $6,974 \pm 0,017$ no & $2,381 \pm 0,002 \mathrm{~lm}$ & $0,033 \pm 0,00008$ no \\
\hline M3 & $1,54 \pm 0,0004 \mathrm{qr}$ & $2,008 \pm 0,010 \mathrm{u}$ & $7,704 \pm 0,036 \mathrm{q}$ & $2,538 \pm 0,046 \mathrm{~m}$ & $0,034 \pm 0,0004$ no \\
\hline M5 & $1,235 \pm 0,006 \mathrm{n}$ & $1,532 \pm 0,006 \mathrm{p}$ & $4,546 \pm 0,037 \mathrm{k}$ & $1,298 \pm 0,046 \mathrm{i}$ & $0,013 \pm 0,0001 \mathrm{defgh}$ \\
\hline
\end{tabular}


Tableau 3: Corrélation(R) entre les différentes méthodes

\begin{tabular}{ccccc}
\hline & TEAC & DPPH & FRAP & RFC \\
\hline DPPH & 0,964 & & & \\
FRAP & 0,939 & 0,934 & & \\
RFC & 0,917 & 0,923 & 0,973 & \\
pH-diff. & 0,404 & 0,417 & 0,364 & 0,443 \\
\hline
\end{tabular}

\section{Teneurs en polyphénols totaux (TPP)}

Ces trois méthodes utilisées pour évaluer les teneurs en antioxydants tendent toutes à montrer qu'un certain nombre de variétés sont homogènes du point de vue de leur teneur en antioxydants tandis que les autres révèlent des différences statistiquement significatives $(\mathrm{P}<0,001)$. De nombreux travaux scientifiques contribuent à montrer que l'activité antioxydante des extraits de plantes est largement due à la présence de certains micronutriments tels que les polyphénols en général et des anthocyanes en particulier. Les bonnes corrélations entre les teneurs en polyphénols et les teneurs en antioxydants évaluées par les trois méthodes précédentes confirment les données de la littérature (Tableau 3, Figure 4). Les teneurs en polyphénols totaux varient de 0,046 pour les variétés KVS411, KVS358, KVS415, KVS416, KVS199, KVS128 et KVS202 à $4,536 \mathrm{mg} \mathrm{d}$ 'EAG/g de graines sèches pour KVS225, KVS97 et KVS312 soit une variation de près de 98 fois.

\section{Teneurs en anthocyanes totales (TAT)}

Les teneurs en anthocyanes totales évaluées sont très faibles dans la majorité des extraits des variétés de voandzou étudiées. Leur teneur est certainement liée à la couleur du péricarpe ; certains étant plus colorés que les autres. Ainsi les quatre variétés qui se distinguent par la couleur pourpre ou noire de leur péricarpe possèdent les teneurs les plus élevées en anthocyanes totales (Tableau 1 et Figure 5). Ce sont D3 < KVS350 < M4 < KVS97, de TAT respectives 0,$139 ; 0,161$; 0,281 et $0,331 \mathrm{mg} / \mathrm{g}$ de graines sèches $(\mathrm{P}<0,001)$.

\section{DISCUSSION}

Les analyses systématiques sur les 44 variétés de voandzou collectées au Burkina
Faso et au Tchad montrent qu'il y a une large variation entre les teneurs en antioxydants, en polyphénols et en anthocyanes totaux.

En dehors des travaux d'isolement, de séparation et d'identification de trois anthocyanines monoglucosides de la delphinidine, la pétunidine et de la malvidine (Eloi et al., 1997), nous n'avons pas connaissance d'une étude comparative des teneurs en antioxydants de plusieurs variétés de $V$. subterranea en relation avec leurs teneurs en polyphénols. En revanche, une étude comparative des TAO a été menée sur des légumineuses de la même famille des Fabaceae (Dong et al., 2007) que le $V$. subterranea, montre que Vicia fabalfaba vulgaris possède la plus haute teneur en antioxydants totaux de $1,86 \mathrm{mmol} / 100$ $\mathrm{g}$ (déterminée par la méthode FRAP); ce qui correspond à 4,65 mg d'ET/g de graines sèches. Elle est suivie par Phaseolus vulgaris (niébé) >Glycine maximus (soja) >Vigna unguiculata (niébé) >Lens culinaris (lentille) >Phaseolus vulgaris ssp.>Vigna radiata> dont les TAO correspondantes sont respectivement : 2,$85 ; 2,05 ; 1,62 ; 1,22$; 0,95 et $0,87 \mathrm{mg}$ d'ET/g de graines sèches (Bente et al., 2002). Les variétés KVS411, KVS358, KVS202, KVS415, KVS199, KVS416 possédant une TAO moyenne de $0,214 \mathrm{mg} \mathrm{d}$ 'ET/g de graines sèches sont inferieures à ces graines de la même famille. Cependant, les variétés KVS141, KVS109, KVS225, KVS311, KVS360, KVS97, KVS153, KVS288, KVS312, KVS67, M4, $\mathrm{D} 2, \mathrm{M} 7, \mathrm{M} 2$ et M3 possèdent des TAO plus élevées (> $6 \mathrm{mg}$ d'ET/g de graines sèches) que celle de Vicia faba/faba vulgaris $(4,65 \mathrm{mg}$ d'ET/g) comparable aux variétés KVS350, KVS314 et KVS32 (environ 5 mg d'ET/g). 


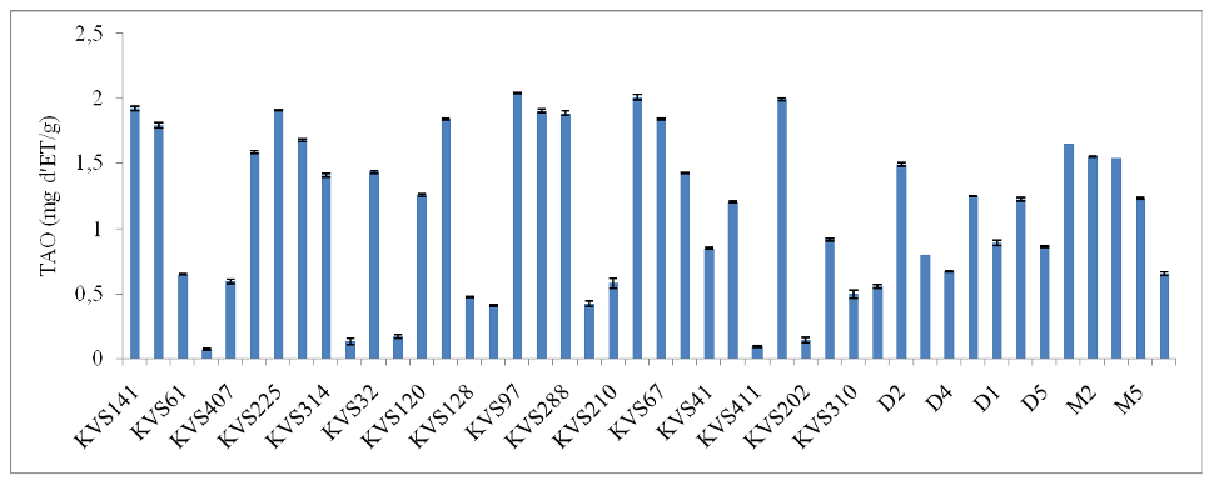

Figure 1: Histogramme comparatif des TAO des différentes variétés des graines de $V$. subterranea (DPPH).

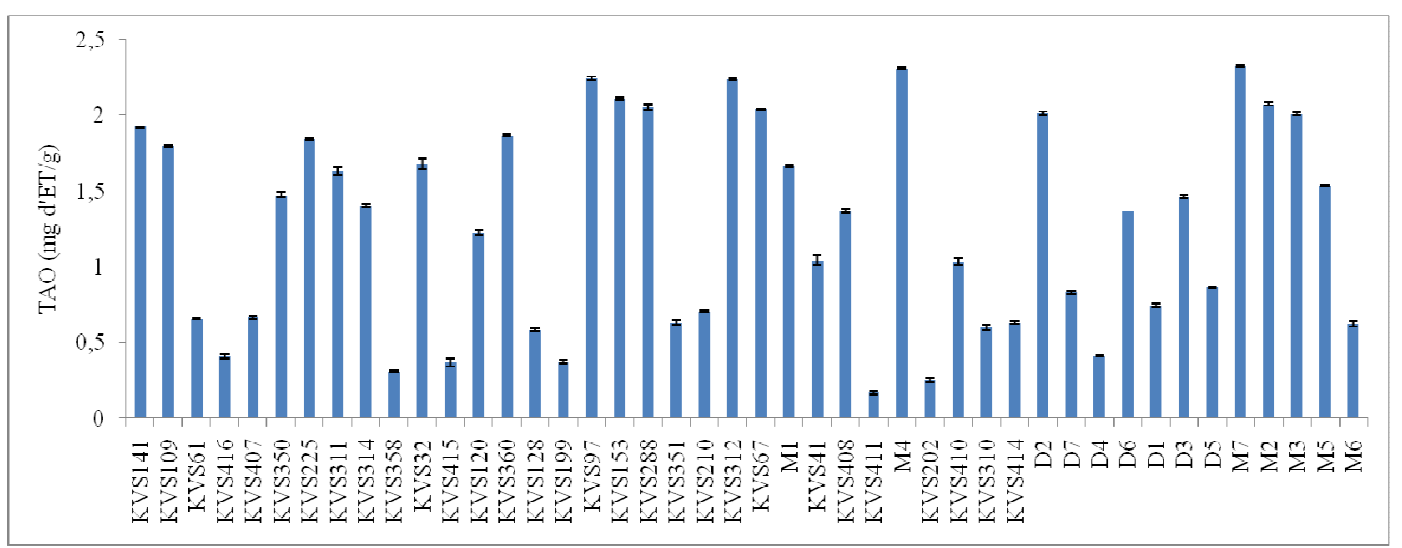

Figure 2: Histogramme comparatif des TAO des différentes variétés des graines de $V$. subterranea (TEAC).

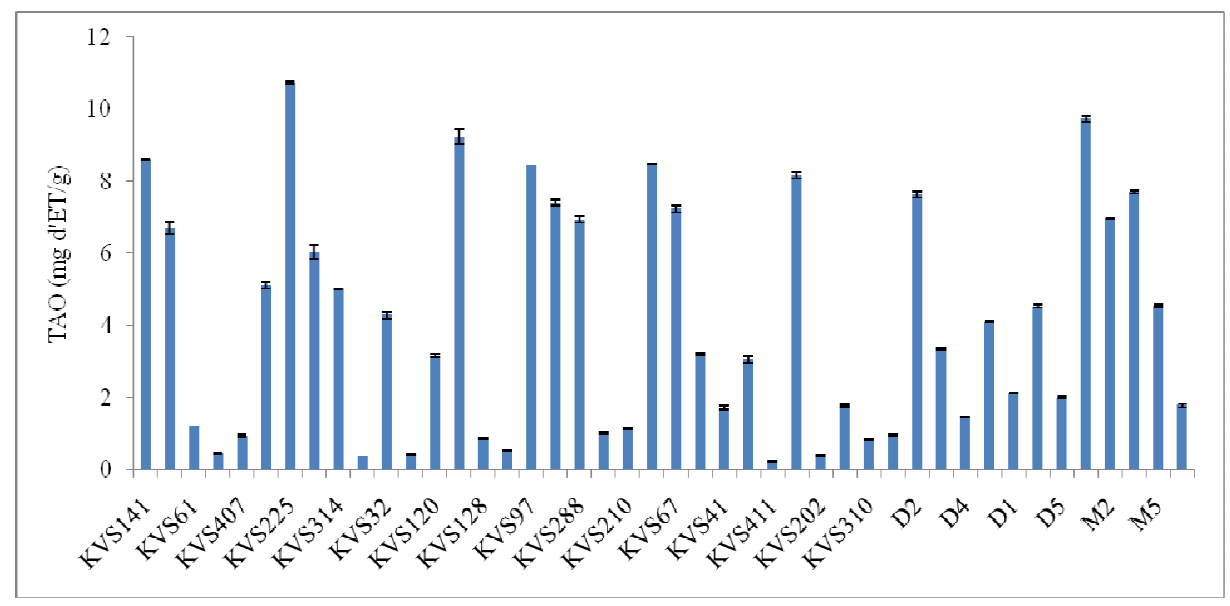

Figure 3: Histogramme comparatif des TAO des différentes variétés des graines de $V$. subterranea (FRAP). 


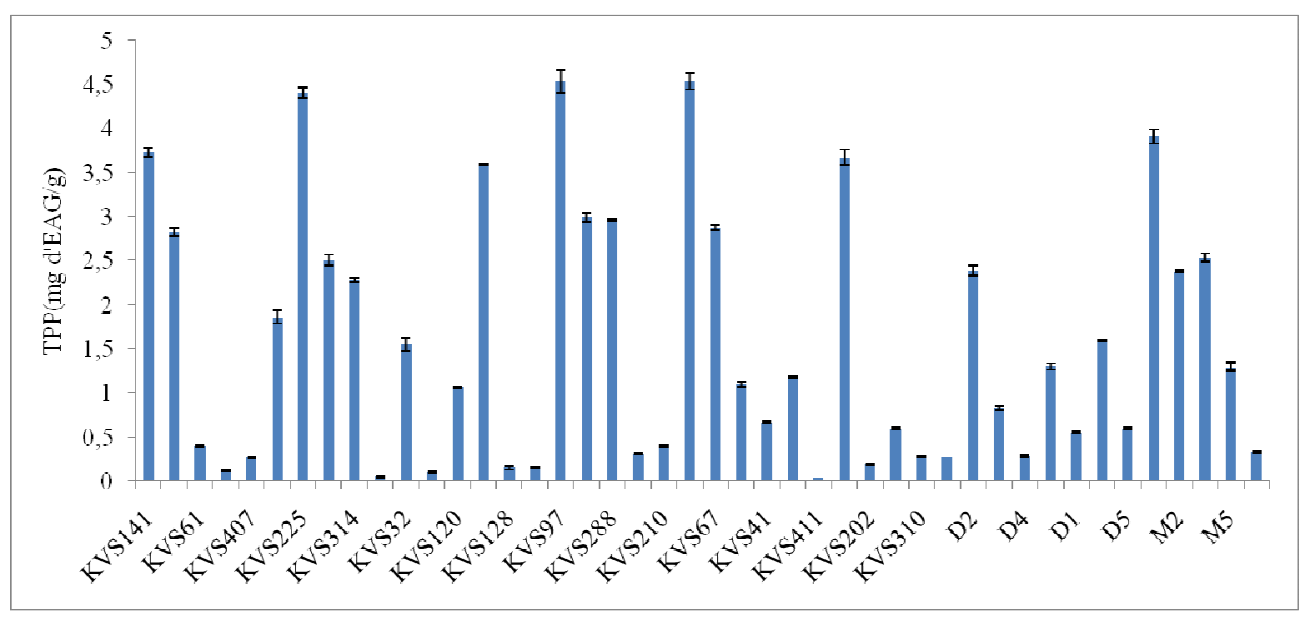

Figure 4: Histogramme des résultats de mesure TPP dans les différentes variétés des graines de $V$. subterranea.

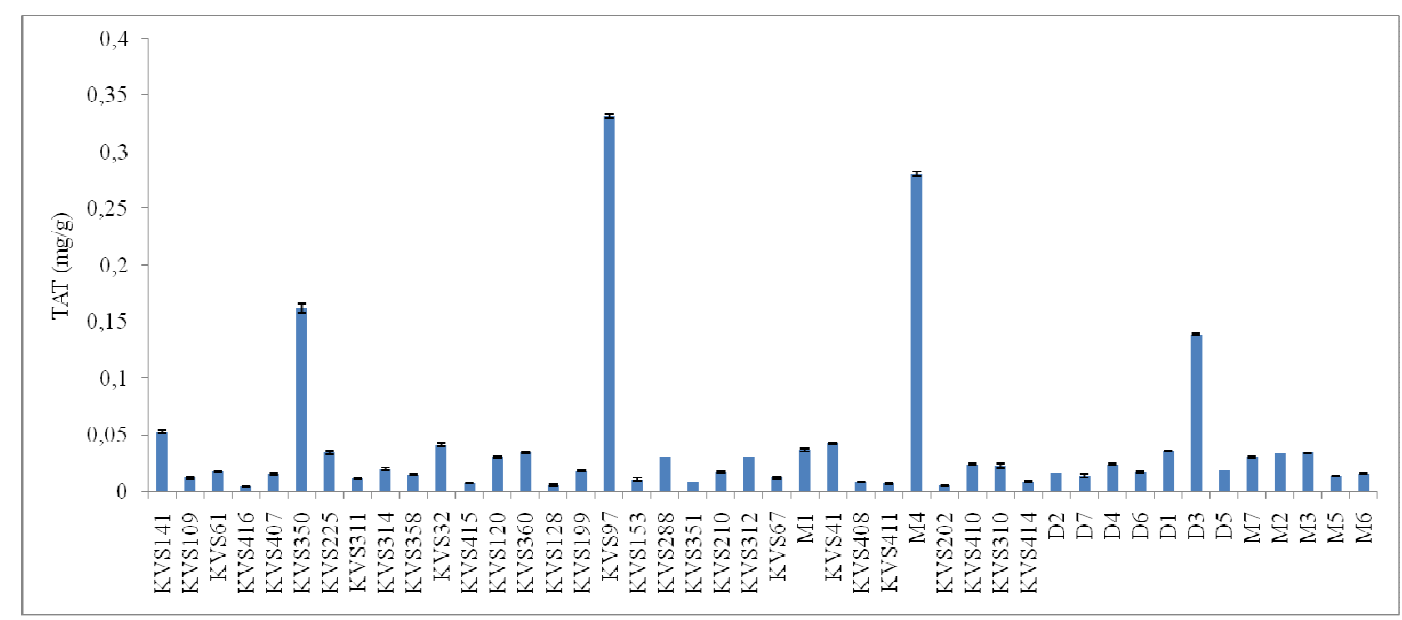

Figure 5: Histogramme des résultats de mesure des TAT des différentes variétés des graines de $V$. subterranea.

La couleur du péricarpe des variétés de $V$. subterranea explique certainement en partie les différences observées entre ces voandzou étudiées. Il est connu que les polyphénols sont responsables à travers des phénomènes de copigmentation intra- ou intermoléculaire (Wigand et al., 1992 ; Leland et al., 2006), de la diversité de couleurs observées (rouge, bleu, mauve, pourpre, etc.) au niveau des feuilles, racines, tiges, écorces, fruits et fleurs des plantes
(Kouda-Bonafos et al., 1994 ; Eloi et al., 1997). De plus, ils sont presqu'entièrement responsables des activités antioxydantes des extraits botaniques (Ou et al., 2005 ;Wojdylo et al., 2007) ; ceci a été confirmé dans cette étude à travers les bons coefficients de corrélation $(\mathrm{r} \sim 0,94)$ observés entre les TPP et les TAO (Tableau 3). En effet, il a été mis en évidence que les polyphénols contribuent à près de $94 \%$ aux teneurs en antioxydants. C'est ce qui explique que les variétés de $V$. 
subterranea les plus colorées surtout en rouge, noir ou plus ou moins bigarrées, présentent les TAO les plus élevées. Un sous-groupe de polyphénols bien connu est celui des anthocyanes caractérisées par leur couleur rouge en milieu acide et bleu en milieu basique (Eloi, 1998), illustrent l'effet de la couleur du péricarpe sur les teneurs en polyphénols et par conséquent sur les teneurs en antioxydants totaux. Parmi les 44 variétés étudiées, il y a seulement les quatre variétés D3, KVS350, M4 et KVS97 dont les teneurs en TAT sont respectivement appréciables : 0,$139 ; 0,161 ; 0,281$ et $0,331 \mathrm{mg} / \mathrm{g}$ de graines sèches. Exceptée D3, ces quatre variétés font partie des 17 variétés possédant un minimum de TAO de $5 \mathrm{mg}$ d'ET/g de graines sèches. Ceci montre que la couleur à elle seule ne saurait expliquer la TAO d'un extrait botanique qui est en réalité un mélange très complexe (de divers composés) (Dejian et al., 2005) du fait que les systèmes de solvant d'extraction ne sont pas toujours spécifiques; ce qui est un facteur de sous- ou de surestimation dans les dosages.

Ainsi, l'étude menée sur les quarante quatre (44) variétés de graines de $V$. subterranea a permis d'identifier KVS350, KVS314, M2, D2, KVS311, M3, KVS109, KVS67, KVS288, KVS153, KVS360, M4, KVS141, M7, KVS225, KVS97, KVS312 présentant une teneur en antioxydants totaux minimum de $6 \mathrm{mg}$ d'ET/g de graines sèches par la méthode FRAP. Cette méthode et les deux autres (DPPH, TEAC), convergent à montrer que le coefficient de corrélation entre la teneur des extraits des graines de $V$. subterranea en polyphénols et l'activité antioxydante est fortement significatif (environ $\mathrm{R}=0,94$ ) ; ce qui indique que $94 \%$ de la capacité antioxydante de ces extraits est due à la contribution des composés phénoliques. Ces variétés sus-citées possèdent des teneurs en polyphénols totaux comparables à celles de sept variétés de sorgho blancs du Sénégal (Khady, 2010).

La teneur en anthocyanes totales est très faible pour la majorité des variétés étudiées, cette teneur étant liée à la couleur du péricarpe ; certaines étant plus colorées que d'autres. Ainsi les quatre variétés qui se distinguent par la couleur pourpre ou noire de leur péricarpe présentent les plus fortes teneurs en anthocyanes; ce sont: D3, KVS350, M4 et KVS97.

Les graines de voandzou ne sont plus seulement perçues comme une source protéino-énergétique mais aussi comme une source de substances phytochimiques «secondaires» dites «antioxydants » et possédant des effets bénéfiques sur la santé des consommateurs. Dans les pays au sud du Sahara, ces légumineuses peuvent donc entrer dans la formulation d'aliments locaux fonctionnels pour la prévention des maladies liées au stress oxydant.

\section{REMERCIEMENTS}

Les auteurs remercient l'Université de Ouagadougou, le CNRST/INERA (Burkina Faso) et l'ITRAD (Tchad) pour tous les efforts consentis dans la réalisation de cette étude. Ils remercient par ailleurs "The MCKNIGHT Foundation" pour le financement de ce travail.

\section{REFERENCES}

Asami KD, Yun-Jeong H, Diane MB, Alyson EM. 2003. Comparison of the total phenolic and ascorbic acid content of freeze-dried marionberry, strawberry, and corn grown using conventional, organic, and sustainable agricultural practices. $J$. Agric. Food Chem., 51: 1237-1241.

Athamena S, Chalghem I, Kassah-Laouar A, Laroui S, Khebri S. 2010. Activité antioxydante et antimicrobienne d'extraits de Cuminum cyminum L. Lebanese Science Journal, 11(1) : 69-81.

Bente LH, Kari H, Mari CWM, Ingrid B, Erlend H, Siv FR, Anne-Brit W, Karin H, Halvard B, Lene FA, Jan M, David RJ, Rune B. 2002. A systematic screening of total antioxidants in dietary plants. $J$. Nutr., 132: 461-471.

Benzie IF, Strain JJ. 1996. The ferric reducing ability of plasma (FRAP) as a measure of 
"antioxidant power": the FRAP assay. Anal. Biochem., 239 : 70-76

Brand-Williams W, Cuvelier ME, Berset C. 1995. Use of free radial method to evaluate antioxidant capacity.Lebensm. Wiss.-Technol., 28: 25-30.

Brink M, Grubben GJH, Brink M, Belay GA. 2006. Ressources Végétales de l'Afrique Tropicale 1: Céréales et Légumes Secs. Edition M. Brink.

Dejian H, Boxin O, Ronald LP. 2005. The chemistry behind antioxidant capacity assays. J. Agric. Food Chem., 53: 18411856.

Dong M, He XJ, Lieu RH. 2007. Phytochemicals Black Bean Seed Coats; Isolation, Structure Elucidation and Their Antiproliferative and Antioxidative Activities. Journal of Agricultural and Food Chemistry, 15: 6044-6051.

Eloi P, Kouda-Bonafos M, Mouhoussine N, Maurice V, Rénée V, Ottinger R. 1997. 7O-methylapigeninidin, an anthocyanidin from Sorghum caudatum. Phytochemistry, 45: 1091-1092.

Eloi P, Mouhoussine N, Maurice V, Renée V. 1997. Anthocyanins from Bambara groundnut (Vigna subterranea). J. Agric. Food Chem., 45: 3359-3361.

Heller J, Begemann F, Mushonga J, 1997. Bambara groundnut Vigna subterranean (L.)Verdc. Conservation and improvement of Bambara groundnut (Vigna subterranea (L.)Verdc.). International Plant, Harare, Zimbabwe.

Jimaima L, Craige T, Mark W, Naiyama W, Subramanium S, Robert P. 2007. Phytochemical flavonols, carotenoids and the antioxidant properties of a wide selections of Fijian fruits, vegetables and other readly available foods. Food Chemistry, 101(4): 1319-1326.

Khady Ba, 2010. Étude comparative des composés phénoliques, du pouvoir antioxydant de différentes variétés de sorgho sénégalais et des enzymes amylolytiques de leur malt. Biotechnol. Agron. Soc. Environ., 14(1) : 131-139.
Kouda-Bonafos M, Czyzewska E, Nacro M. 1994. Isolation of apigeninidin from leaf sheaths of Sorghum caudatum. Journal of Chemical Ecology, 20: 2123-2125.

Leland JC, Kirakosyan A, Kaufman PB, Warber SL, Duke JA, Brielmannk HL. 2006. Natural Products from Plants (2nd edn), CRC Press.

Liangli LY, Kequan KZ, John P. 2005. Antioxidant properties of cold-pressed black caraway, carrot, cranberry, and hemp seed oils. Food Chemistry, 91: 723-729.

Liu RH. 2003. Health benefits of fruits and vegetables are from additive and synergistic combination of phytochemicals. Am. J. Clin. Nutr., 78: 517-520.

Mahama O, Jeremy TO, Jean Baptiste T, Didier B, Clémentine BD, Gnissa K. 2008. Characterization and evaluation of accessions of Bambara groundnut (Vigna subterranean (L.) Verdcourt) from Burkina Faso. Sci. Nat., 5(2): 191-197.

Minka DR, Bruneteau M. 2000. Partial chemical composition of bambara pea (Vigna subterranean L. Verdc.). Food Chem., 68: 273-276.

Navindra PS. 2008. Berry Fruits: Compositional Elements, Biochemical Activities, and the Impact of Their Intake on Human Health, Performance, and Disease. J. Agric. Food Chem., 56: 627629.

Nihal TY, Sedat V, Ferda S, Gokce P. 2007. Effect of Extraction Conditions on Measured Total Polyphenol Contents and Antioxidant and Antibacterial Activities of Black Tea. Molecules, 12: 484-496.

$\mathrm{Ou}$ B, Prior RL, Huang D. 2005. The chemistry behind dietary antioxidant capacity assays. J. Agric. Food Chem., 53: 1841-1856.

Palé E. 1998. Contribution à l'étude des composés anthocyaniques des plantes: cas de Hibiscus sabdariffa, Lannea microcarpa, Vigna subterranea et Sorghum caudatum du Burkina Faso. 
Thèse de troisième Cycle, Université de Ouagadougou, Ouagadougou, p. 16-17.

Pellegrini N, Del Rio D, Colombi B, Bianchi M, Brighenti F. 2003. Application of the 2,2'-azinobis (3-ethylbenzothiazoline-6sulfonic acid) radical-cation assay to a flow injection system for the evaluation of antioxidant activity of some pure compounds and beverages. J. Agric. Food Chem., 51: 260-264.

Proteggente AR, Pannala AS, Paganga G, Van Buren L, Wagner E, Wiseman S, Van De Put F, Dacombe C, Rice-Evans CA. 2002. The antioxidant activity of regularly consumed fruit and vegetables reflects their phenolic and vitamin $\mathrm{C}$ composition. Free Radical Res., 36(2): 217-33.

Sellapan S, Akoh CC. 2002. Flavonoids and antioxidant capacity of Georgia-grown blueberries and blackberries. J. Agric. Food Chem., 50: 2432-2438.

Stephanie D, Xavier V, Philippe C, Marion W, Jean-Michel M. 2009. Comparative
Study of Antioxidant Properties and Total Phenolic Content of 30 Plant Extracts of Industrial Interest Industrial Interest Using DPPH, TEAC, FRAP, SOD, and ORAC Assays. J. Agric. Food Chem., 57: 1768-1774.

Wigand MC, Dangles O, Brouillard R. 1992. Complexation of a fluorescent anthocyanin with purines and polyphenols. Phytochemistry, 31(12): 4317-4324.

Wojdylo A, Oszmianski J, Czemerys R. 2007. Antioxidant activity and phenolic compounds in 32 selected herbs. Food Chem., 105: 940-949.

Yanjun Z, Navindra PS, Rupo L, Lydia F, David H. 2008. Isolation and identification of strawberry phenolics with antioxidant and human cancer cell antiproliferative properties. J. Agric. Food Chem., 56: 670-675. 